\title{
Multiphoton Microscopic Study of the Renal Cell Carcinoma Pseudocapsule: Implications for Tumour Enucleation
}

\section{Yi Quan Tan}

National University Health System https://orcid.org/0000-0001-5303-4237

\section{Wy Keat Tay}

National University Health System

Li Yin Ooi

National University Health System

Thamboo Thomas Paulraj

National University Health System

Ho Yee Tiong ( $\nabla$ surthy@nus.edu.sg )

https://orcid.org/0000-0003-0077-7904

\section{Research article}

Keywords: Renal Cell Cancer, Nephrectomy, Multiphoton Fluorescence Microscopy, Surgical Margins, Tumour Enucleation

Posted Date: March 31st, 2020

DOl: https://doi.org/10.21203/rs.3.rs-18386/v1

License: (c) (i) This work is licensed under a Creative Commons Attribution 4.0 International License. Read Full License

Version of Record: A version of this preprint was published at Urology on October 1st, 2020. See the published version at https://doi.org/10.1016/j.urology.2020.06.064. 


\section{Abstract}

Background To utilise Multiphoton Microscopy as a novel imaging technique to characterize and quantify collagen at the Renal Cell Carcinoma Pseudocapsule, to assess for both intra-tumoral and inter-tumoral variation of collagen characteristics. MPM combines Second Harmonic Generation and Two Photon Excitation Fluorescence to image extracellular matrix architecture.

Methods 20 partial nephrectomy specimen tissues were retrieved, cut into 5-micron sections, mounted on slides and deparaffinized. The pseudocapsules were imaged with $2 \mathrm{X}$ and $20 \mathrm{X}$ objective at selected Regions of Interest. Corresponding clinical information was retrieved. Pseudocapsule thickness was determined. Collagen parameters measured included quantification by the Collagen Area Ratio, and qualitative measurements by the Collagen Fiber Density and Collagen Reticulation Index.

Results The boundaries between tumor, PC and normal renal parenchyma were distinguished by Multiphoton Microscopy without the need for staining. In the thickest areas of the pseudocapsule, collagen content and density were quantitatively higher compared to the thinnest areas. Median Collagen Area Ratio was higher in the thickest compared to the thinnest areas of the PC $(p=0.01)$. Clear Cell RCC specimens had a consistently higher Collagen Fiber Density in both the thickest and thinnest areas compared to non-Clear Cell RCC specimens $(p=0.02)$.

Conclusions In this study, we demonstrated the ability of Multiphoton Microscopy to quantify collagen characteristics of pseudocapsules without fluorescent labelling. Tumor enucleation for Renal Cell Carcinoma along its Pseudocapsule remains debatable with regards to oncological safety. Even with a complete and intact pseudocapsule, the pseudocapsule is not a homogenous structure, and varies in its thickness and its collagen characteristics within, and between, tumours.

\section{Introduction}

Recent studies have demonstrated the importance of the Pseudocapsule (PC) around the Renal Cell Carcinoma (RCC) tumor as a boundary between tumor and normal renal parenchyma. PC integrity has been shown to have prognostic significance, with worse long term overall and progression-free survival for tumors lacking a PC or having PC invasion. ${ }^{1}$ The recognition of the importance of PCs led to a proposed standardized PC invasion scoring system to classify PC integrity, as current standard pathological reporting do not routinely report PC integrity. ${ }^{2} \mathrm{~A}$ major component of the fibrous PC is collagen. ${ }^{3}$ Collagen, being an abundant Extra-Cellular Matrix (ECM) component, is the strongest and hardest to penetrate by tumor cells. ${ }^{4}$ During tumor expansion, collagen remodeling and reorientation occurs, with increased collagen deposition and density for mechanical strength to resist tumor growth. Degradation of collagen has also been shown necessary for tumor invasion. ${ }^{4}$ However, limited literature exists on the collagen characteristics of PCs, which are at the interface of tumor expansion, and probably important in resisting invasion. 
MPM combines Second Harmonic Generation (SHG) and Two Photon Excitation (TPE) Fluorescence to image extracellular matrix architecture in great detail. ${ }^{5}$ Although MPM has been used to image kidney tumors, MPM's capabilities are in the detailed evaluation of collagen and is potentially a better tool to evaluate collagen characteristics at the tumor-parenchymal boundary.

Tumor Enucleation for RCC has gained popularity as a Nephron-Sparing Surgery technique to maximally preserve renal parenchyma and reduce ischemia time. ${ }^{6}$ Tumor Enucleation relies on dissection along the fibrous Pseudocapsule at the tumor-parenchyma interface. Hence, a deeper understanding of the inherent characteristics of the PC as a tumor-parenchyma boundary is important to determine the safety of Tumor Enucleation as an oncological approach. ${ }^{7}$ Therefore, the aim of this study was to utilize MPM as a novel imaging technique to characterize and quantify collagen at the PC, and assess for both intra-tumoral and inter tumoral variations in the characteristics of the PC.

\section{Materials And Methods \\ 2.1 Sample Acquisition}

The study protocol was approved by the National Healthcare Group Domain Specific Review Board, Reference Number: 2017/01003. From the Ethics Board-approved tissue repository, 20 standard partial nephrectomy specimen tissue blocks from our Centre were selected and retrieved. All patients gave consent for the study. All 20 specimens were from standard on-clamp partial nephrectomies performed for PT1 malignant tumors from 2017-2018. Tissue samples from three RCC histological subtypes according to the 2004 WHO Classification ${ }^{8}$ were chosen (clear cell, papillary and chromophobe RCC) by a single institutional pathologist. For the purposes of this study, all 20 specimens selected had complete PCs with no evidence of tumour invasion, and had margins of normal renal tissue on the parenchymal interface. For each specimen, paraffin tissue blocks which contained the tumour-parenchymal interface were retrieved. Tumor grade was determined according to the Fuhrman Grading System. ${ }^{9}$ Tumor stage was based on the updated 8th Edition American Joint Committee on Cancer Staging Manual. ${ }^{10}$ Nonidentifiable corresponding clinical information from our partial nephrectomy database was retrieved.

\subsection{Sample Preparation and Multi-Photon Microscopy}

The unstained de-paraffinized Formalin-Fixed Paraffin-Embedded tissues of 5-micron thickness were imaged using the commercially available laser-based Genesis200 ${ }^{\text {Tm }}$ Multi-Photon Microscopy system (Histoindex Pte. Ltd, Singapore). Image acquisition was performed at $2 \mathrm{X}$ and $20 \mathrm{X}$ objective with $512 \times$ 512-pixel resolution (Fig. 1). MPM is an imaging modality comprising SHG and TPE microscopy. SHG signals are detected when a laser of femtosecond range interacts with tissues that have unique, noncentrosymmetric structure within the ECM such as collagen fibers and elastin. When this SHG signal is combined with auto-fluorescence signal from the cells using TPE, MPM is able to penetrate deep into tissues to generate a high-resolution image that reflects both the architecture of the ECM and surrounding cellular structure. ${ }^{11}$ On SHG microscopy, the fibrous PC was readily identified, with fluorescent green 
signals on SHG analysis corresponding to collagen fibers (Fig. 2). In contrast, collagen-devoid tumor tissues and collagen-poor normal renal parenchyma demonstrated minimal SHG signals.

Embedded within the proprietary stain-free imaging technology and analysis software lies the capability to detect sensitively and quantify fine collagen dynamics that are otherwise unobserved with traditional staining techniques. ${ }^{11}$ The analysis is able to identify and characterize previously described SHG collagen characteristics that corresponds with the collagen profiles of the tissues. ${ }^{12-14}$ The area occupied by collagen relative to the total area of the sample was expressed as the Collagen Area Ratio (CAR). The density of collagen fibers within a total given area was expressed as the Collagen Fiber Density (CFD). Both CAR and CFD quantify the collagen content of the tissues. After the collagen area was defined, a skeletonization of the collagen was applied, providing a schematic representation of the collagen fibers and their intersection points. The Collagen Reticulation Index (CRI) was defined as the total number of intersection points in the given collagen area, representing the degree of reticulation and interconnection of collagen fibers.

Imaging and analysis were performed for the PC regions abutting the adjacent normal parenchymal tissue for each of the specimens. The thickest and thinnest PC areas for each tumour specimen were designated Regions of Interests (ROIs) for PC analysis of collagen parameters in each specimen. At each $\mathrm{ROI}$, clinically validated algorithms quantify collagen content and structure. These algorithms were validated against conventional stained H\&E images by pathologists at the National University Hospital, Singapore. Collagen content was measured quantitatively by CAR and CFD, and collagen structure was measured by CRI.

\subsection{Statistical Analysis}

Parametric continuous variables were reported as Mean +/- Standard Deviation. Non-parametric continuous variables were reported as Median (Inter-Quartile Range). There were no missing values from data collected, and there were no side effects to the patients. The Mann-Whitney $U$ test was used to compare non-parametric continuous variables. Statistical significance in this study was set as $p<0.05$. All reported P values were 2-sided, and analyses were performed with SPSS (version 17.0).

\section{Results}

\subsection{Baseline Characteristics}

Baseline patient demographics and tumor characteristics are shown in Table 1. 20 RCC specimens (11 clear cell, 6 papillary, 3 chromophobe) were imaged. 15 patients were male, and mean age was $60.6+/-$ 10.6 years. Mean tumor size was $3.32+/-1.47 \mathrm{~cm}$. There were 5 low, 13 medium, and 2 high complexity tumors on RENAL Nephrometry score. 14 were pathological stage T1a tumors while 6 were T1b tumors. Among the 11 clear cell RCC specimens, 8 were Fuhrman Grade 3-4 tumors. 
Table 1

Patient Demographics and Tumor Characteristics

\begin{tabular}{|ll|}
\hline Number of Tumors & $\mathbf{2 0}$ \\
\hline Mean Age (years) & $60.6+/-10.6$ \\
\hline Number of Males (\%) & $15(75)$ \\
\hline Mean Tumor Size (cm) & $3.32+/-1.47$ \\
\hline Right Sided Tumors (\%) & $11(55)$ \\
RENAL Nephrometry Score (\%): & $5(25)$ \\
$4-6$ (low) & $13(65)$ \\
$7-9$ (moderate) & $2(10)$ \\
10-12 (high) & \\
\hline Tumor Histological Subtype (\%): & $11(55)$ \\
Clear Cell & $6(30)$ \\
Papillary & $3(15)$ \\
Chromophobe & \\
\hline Fuhrman Grade for Clear Cell Tumors (\%): & $3(27.2)$ \\
1-2 & $8(72.7)$ \\
3-4 & \\
\hline Pathological TNM Stage (\%): & $14(70)$ \\
T1a & $6(30)$ \\
T1b & $0(0)$ \\
T2-4 & \\
\hline Partial Nephrectomy Surgical Approach (\%): & $5(25)$ \\
Open & $3(15)$ \\
Laparoscopic & $12(60)$ \\
Robotic-Assisted Laparoscopic & \\
\hline
\end{tabular}

\subsection{Pseudocapsule MPM Imaging of RCC Histological Subtypes}

The boundaries between tumor, $\mathrm{PC}$ and normal renal parenchyma were distinguished without the need for staining under light microscopy (Fig. 1). Figure 4 shows the imaging and tumor specimen of a pT1a Papillary RCC specimen with a RENAL Nephrometry score of 6 a. Figure 5 shows the imaging and tumor specimen of a pT1b Clear Cell RCC specimen with a RENAL Nephrometry score of 9p. Figures 4 and 5 highlights clearly the wide differences in the pseudocapsules of both tumors, not just in terms of thickness and continuity but also the fiber deposition pattern and density of the fibers.

\subsection{Pseudocapsule Collagen Characteristics}

The median thickness of the thickest areas of the PCs was $0.5(0.3-0.7) \mathrm{mm}$, compared to $0.2(0.1-0.3)$ $\mathrm{mm}$ in the thinnest areas. In the thickest areas of the PC, collagen content and density were quantitatively higher compared to the thinnest areas (Table 2). Median CAR was significantly higher in the thickest 
compared to the thinnest areas of the PC $(p=0.01)$. Mean CFD was also higher $(p=0.09)$ in the thickest compared to the thinnest areas of the PC $(p=0.09)$. The thickest areas of the PCs showed measurable higher degrees of collagen cross-linking and collagen network complexity. Mean CRI was higher in the thickest PC areas compared to the thinnest areas of the PC $(p=0.19)$.

Table 2

Collagen Characteristics of Pseudocapsules within each tumor (Intra-tumor variation)

\begin{tabular}{|llll|}
\hline & $\begin{array}{l}\text { Thickest PC Areas } \\
\text { (ROI1) }\end{array}$ & $\begin{array}{l}\text { Thinnest PC Areas } \\
\text { (ROI2) }\end{array}$ & $\begin{array}{l}\text { p- } \\
\text { value }\end{array}$ \\
\hline PC Thickness (mm) & $0.5(0.3-0.7)$ & $0.2(0.1-0.3)$ & 0.001 \\
\hline Median Collagen Area Ratio (\%) & $24.3(20.7-36.1)$ & $20.2(15.0-22.4)$ & 0.01 \\
\hline $\begin{array}{l}\text { Median Collagen Fiber Density (\%) } \\
\begin{array}{l}\text { Median Collagen Reticulation } \\
\text { Index }\end{array}\end{array}$ & $36.0(22.7-54.3)$ & $27.1(21.4-36.0)$ & 0.09 \\
\hline
\end{tabular}

Table 3 and Table 4 shows the comparison between PC collagen characteristics between the thickest and thinnest areas of the PC, stratified by histological subtype, RENAL Nephrometry score and tumor size. Clear Cell RCC specimens had a consistently higher collagen fiber density in both the thickest and thinnest areas compared to non-Clear Cell RCC specimens $(p=0.02)$. There were no other statistically significant differences when comparing the other subgroups. 
Table 3

Comparison of Thickest PC areas between tumors $(n=20)$ based on Histological Subtype, Nephrometry Score and Tumor Size

\begin{tabular}{|c|c|c|c|}
\hline Histology & Clear Cell $(n=11)$ & Non-Clear Cell $(n=9)$ & $\begin{array}{l}\mathrm{p}- \\
\text { value }\end{array}$ \\
\hline $\begin{array}{l}\text { Median Collagen Area Ratio } \\
(\%)\end{array}$ & $28.8(23.0-36.3)$ & $31.3(18.4-46.2)$ & 0.30 \\
\hline $\begin{array}{l}\text { Median Collagen Fiber } \\
\text { Density (\%) }\end{array}$ & $47.7(31.9-59.5)$ & $28.5(30.1-38.1)$ & 0.02 \\
\hline $\begin{array}{l}\text { Median Collagen } \\
\text { Reticulation Index }\end{array}$ & $3.96(3.23-4.86)$ & $3.38(2.63-3.95)$ & 0.15 \\
\hline Complexity & $\begin{array}{l}\text { Low Nephrometry Score } \\
(n=5)\end{array}$ & $\begin{array}{l}\text { Moderate-high Nephrometry } \\
\text { Score }(n=15)\end{array}$ & $\begin{array}{l}\mathrm{p}- \\
\text { value }\end{array}$ \\
\hline $\begin{array}{l}\text { Median Collagen Area Ratio } \\
(\%)\end{array}$ & $33.1(23.8-46.2)$ & $28.9(18.6-36.3)$ & 0.29 \\
\hline $\begin{array}{l}\text { Median Collagen Fiber } \\
\text { Density (\%) }\end{array}$ & $37.8(17.4-52.9)$ & $39.5(23.4-58.6)$ & 0.73 \\
\hline $\begin{array}{l}\text { Median Collagen } \\
\text { Reticulation Index }\end{array}$ & $3.17(1.99-4.06)$ & $3.88(3.07-4.86)$ & 0.29 \\
\hline Stage & $T 1 a(n=14)$ & $\mathrm{T} 1 \mathrm{~b}(\mathrm{n}=6)$ & $\begin{array}{l}\mathrm{p}- \\
\text { value }\end{array}$ \\
\hline $\begin{array}{l}\text { Median Collagen Area Ratio } \\
(\%)\end{array}$ & $32.2(22.1-41.7)$ & $24.6(14.6-35.6)$ & 0.48 \\
\hline $\begin{array}{l}\text { Median Collagen Fiber } \\
\text { Density (\%) }\end{array}$ & $36.5(21.9-51.5)$ & $45.0(28.1-61.3)$ & 0.30 \\
\hline $\begin{array}{l}\text { Median Collagen } \\
\text { Reticulation Index }\end{array}$ & $3.70(2.97-4.32)$ & $3.71(2.61-4.87)$ & 0.97 \\
\hline
\end{tabular}


Table 4

Comparison of Thinnest PC areas between tumors $(n=20)$ based on Histological Subtype, Nephrometry Score and Tumor Size

\begin{tabular}{|c|c|c|c|}
\hline Histology & Clear Cell & Non-Clear Cell & $\begin{array}{l}\mathrm{p}- \\
\text { value }\end{array}$ \\
\hline $\begin{array}{l}\text { Median Collagen Area Ratio } \\
(\%)\end{array}$ & $21.7(16.8-25.2)$ & $21.7(17.9-23.9)$ & 0.48 \\
\hline $\begin{array}{l}\text { Median Collagen Fiber } \\
\text { Density (\%) }\end{array}$ & $34.7(24.1-38.3)$ & $22.8(16.8-27.3)$ & 0.02 \\
\hline $\begin{array}{l}\text { Median Collagen } \\
\text { Reticulation Index }\end{array}$ & $3.50(3.12-4.04)$ & $2.93(2.01-3.42)$ & 0.08 \\
\hline Complexity & $\begin{array}{l}\text { Low Nephrometry } \\
\text { Score }(<7)\end{array}$ & $\begin{array}{l}\text { Mid-high Nephrometry Score (7 } \\
\text { or more) }\end{array}$ & $\begin{array}{l}\mathrm{p}- \\
\text { value }\end{array}$ \\
\hline $\begin{array}{l}\text { Median Collagen Area Ratio } \\
(\%)\end{array}$ & $25.7(13.0-40.3)$ & $20.4(15.7-22.3)$ & 0.99 \\
\hline $\begin{array}{l}\text { Median Collagen Fiber } \\
\text { Density (\%) }\end{array}$ & $21.4(16.1-26.1)$ & $32.0(21.4-38.1)$ & 0.05 \\
\hline $\begin{array}{l}\text { Median Collagen } \\
\text { Reticulation Index }\end{array}$ & $2.93(1.54-4.30)$ & $3.35(2.88-4.00)$ & 0.48 \\
\hline Stage & T1a & $\mathrm{T} 1 \mathrm{~b}$ & $\begin{array}{l}\mathrm{p}- \\
\text { value }\end{array}$ \\
\hline $\begin{array}{l}\text { Median Collagen Area Ratio } \\
(\%)\end{array}$ & $19.81(14.5-21.9)$ & $20.1(14.4-24.5)$ & 0.54 \\
\hline $\begin{array}{l}\text { Median Collagen Fiber } \\
\text { Density (\%) }\end{array}$ & $22.9(19.8-31.8)$ & $34.8(28.1-41.1)$ & 0.09 \\
\hline $\begin{array}{l}\text { Median Collagen } \\
\text { Reticulation Index }\end{array}$ & $3.28(2.62-4.01)$ & $3.17(2.45-3.72)$ & 0.77 \\
\hline
\end{tabular}

\section{Discussion}

This initial study demonstrated that the use of commercially available MPM enabled detailed imaging of the PCs at the tumour-parenchymal interface of RCCs to facilitate precise measurements of its thickness and collagen characteristics. At the tumour-parenchymal interface, there was significant variation in the thickness and hence median collagen area of the PC between the thinnest and thickest regions of interest within each tumour. Clear cell tumours were found to have significantly median collagen fibre densities than non-clear cell tumours at both the thinnest and thickest regions of interest in the PC.

Multi-Photon Microscopy is a highly accurate tool for imaging of collagen and analysing its organization from molecular scale up to tissue architectural level. It combines the advantages of a non-linear imaging 
approach with a coherent modality able to probe molecular organization. ${ }^{12-13}$ Another key advantage is the ability to process fresh, unstained tissues without the need for staining. ${ }^{5}$ Historically, pathologists have focused on the morphological characteristics of cancer cells under light microscopy, such as their architectural growth patterns and cytological features, in the subtyping and grading of tumours. However, with the high sensitivity and specificity in probing collagen characteristics, MPM has increasingly been employed in various cancer tissues. It has been suggested that MPM has the ability to grade, differentiate and prognosticate certain cancers, for example breast, ${ }^{14}$ ovarian $^{15}$ and gastric cancers. ${ }^{16}$

Multi-Photon Microscopy has also been employed in urological cancers. Ling et al found that Gleason grade in Prostate cancer could be correlated with the ratio of anisotropic and isotropic collagen fibres in biopsy tissues imaged with MPM, potentially serving as a biomarker for cancer aggressiveness. ${ }^{17}$ Brooks et al used MPM to investigate collagen structure of non-muscle invasive bladder cancer, demonstrating different collagen fibre morphology in patients with subsequent invasive progression, potentially serving as a prognostication and treatment decision tool. ${ }^{18}$

MPM has been previously used to image RCC tumour specimens, without analysis of the pseudocapsule. Best et al demonstrated higher collagen fibre density and alignment in grade 4 compared to grade 1 RCC, and suggested that MPM could complement traditional tumour grading in future. ${ }^{19}$ In a study by Jain et al, MPM demonstrated ability to differentiate tumor from normal parenchyma in fresh, unstained kidney tissues. In their study, it distinguished RCC histological subtypes based on their tissue architecture, with a diagnostic accuracy of $95 \% .{ }^{20}$ It was suggested that MPM may potentially be used for real time unstained kidney tissue biopsy diagnosis and surgical margin assessment in future. ${ }^{20}$

The findings in our report support the other reports that the presence and integrity of RCC pseudocapsules are known to vary between tumours. Higher rates of PC presence and PC completeness have been found in clear cell RCC. ${ }^{3}$ Higher risk of PC invasion have been demonstrated in larger tumors and tumors with incomplete PC. ${ }^{21} \mathrm{PC}$ invasion has also been associated with higher grade and stage of tumors, which independently predict the risk of tumor recurrence. ${ }^{1}$ Kim et al studied 258 patients with clinical T1a tumours who underwent standard partial nephrectomy. It was found that $26.7 \%$ of tumours had incomplete PCs, $14.3 \%$ had absent PCs, and $17.4 \%$ had complete PC invasion. ${ }^{22}$ However, the reason why certain tumour subtypes and higher grade tumours have incomplete PCs or have higher rates of PC invasion remain poorly understood. Our studies suggest that the PCs in RCCs with clear cell histology may be tougher with significantly greater collagen fibre density. This suggest that RCCs with clear cell histology may have tougher PCs than non-clear cell histology.

Indeed, there are few studies analysing the characteristics of PCs beyond just structural integrity. Provenazano et al described the process of increased extra-cellular matrix collagen deposition, remodelling and crosslinking to resist tumour expansion. ${ }^{23}$ Wang et al studied of PCs in small renal masses, and established collagen and reticulin to be abundant within the fibrous stroma of PCs. Pseudocapsules were shown to be a rim of compressed fibrous tissue at the tumour-parenchymal 
margin. Through special staining with Tri-chrome, they demonstrated consistently high collagen content in PCs across all tumour histological subtypes. ${ }^{24}$ The high collagen content is the reason why MPM is potentially a game-changing tool for providing insights into the characteristics and biological behaviour of PCs. For example, in addition to inter tumour variations, our study showed that the RCC PC has significant thickness and collagen area variation even within each tumour along the tumour-parenchymal interface. Despite the differences in PC thickness, collagen fibre density and the complexity of linkages between the fibres (as measured by the reticulation index) are not significantly different between the thinnest and thickest areas of PC for each tumour. Intriguingly, this suggest that the site of any PC invasion by its tumour is likely a function of the thickness alone and not 'structural' weakness.

This is the first study to employ the stain-free MPM technology to analyse collagen characteristics of RCC pseudocapsules. Our data suggests that each RCC PC is not a consistent layer when fine collagen characteristics are examined using the stain-free MPM technology. Even with a truly complete and intact $\mathrm{PC}$, we have shown that there are quantifiable and demonstrable intra-tumoral variation of collagen quantity and quality within each tumour PC. This has implications on the technique of Tumour Enucleation, which relies on the complete dissection along the fibrous PC. The PC, being the boundary between normal tissue and tumour cells, is a key factor during enucleation and surgeons should be aware of such intra-tumoral variation in PC thickness to achieve precise and complete tumour enucleation. Despite favourable published data, concerns over the oncological safety and positive margin rates of enucleation remain, mainly related to the integrity of the PC. ${ }^{1,22}$ Indeed, intertumoral differences of collagen density between clear and non-clear cell cancers in our study suggest different pseudocapsular toughness when exposed to surgical dissection during enucleation. This may be the reason why anecdotally, surgeons may have avoided enucleation for papillary cell cancers for fear of rupturing the weaker PCs, resulting in tumour spillage.

The main drawback of the study is the small numbers and the retrospective nature. A larger number over a longer study period would enable us to better correlate collagen characteristics with different tumour histological subtypes, grades and stages. Indeed, with increasing use of better imaging techniques, it is anticipated others will utilize MPM to corroborate and extend our initial findings.

\section{Conclusions}

In this study, we have demonstrated the ability of MPM to quantify collagen characteristics of pseudocapsules without fluorescent labelling. It is important for Urologists to be aware that even with a complete and intact pseudocapsule, it is not a homogenous structure, and varies in its thickness and its collagen characteristics within and between tumours.

\section{Abbreviations}

RCC: Renal Cell Carcinoma 
PC: Pseudocapsule

ECM: Extra-Cellular Matrix

MPM: Multiphoton Microscopy

SHG: Second Harmonic Generation

TPE: Two Photon Excitation

\section{Declarations}

Ethics approval and consent to participate: The study protocol was approved by the National Healthcare Group Domain Specific Review Board, Reference Number: 2017/01003. Consent was obtained from all study participants.

Consent for publication: N.A. No identifiable data.

Availability of data and materials: The datasets used and/or analysed during the current study are available from the corresponding author on reasonable request.

Competing interests: The authors declare that they have no competing interests.

Funding: National University Health System Innovation Seed Grant 2017 (intra-institution). There was no extra-institution or commercial funding.

Authors' contributions: YQ was involved in the data analysis and manuscript preparation. WK was involved in the planning of the study. LY and TT were involved the histological preparation of the samples. HY was involved in the planning of the study and manuscript preparation. All authors read and approved the final manuscript.

Acknowledgements: The authors would like to acknowledge Histoindex Pte. Ltd, Singapore and Clinnovate Health Pte. Ltd, Singapore for performing Multiphoton Microscopy of the samples using the Genesis200 ${ }^{\text {мм }}$ system.

Authors' information: N.A.

\section{References}

1. Lu Q, Ji C, Zhao X, et al. Histopathologic analysis of tumour bed and peritumoral pseudocapsule after in vitro tumour enucleation on radical nephrectomy specimen for clinical T1b renal cell carcinoma. Urol Oncol 2017; 35: 603.

2. Snarskis C, Calaway A, Wang L et al. Standardized Reporting of Microscopic Renal Tumour Margins: Introduction of the Renal Tumour Capsule Invasion Scoring System. J Urol 2017; 197: 23. 
3. Jacob S, Williamson S, Gondim D et al. Characteristics of the Peritumoral Pseudocapsule Vary Predictably with Histologic Subtype of T1 Renal Neoplasms. Urology 2015; 86: 956.

4. Xi W, Wang J, Liu L et al. Collagen as a double-edged sword in tumour progression. Tumour Biol 2014; 35: 2871.

5. Cicchi R, Vogler N, Kapsokalyvas D et al. From molecular structure to tissue architecture: collagen organization probed by SHG microscopy. J Biophotonics 2013; 6: 129.

6. Smith Z, Malkowicz S. Tumour Enucleation for Renal Cell Carcinoma. J Kidney Cancer VHL 2015; 2: 64.

7. Azhar R, Abreu A, Broxham E et al. Histological analysis of the kidney tumour-parenchyma interface. J Urol 2015; 193: 415.

8. Lopez-Beltran A, Scarpelli M, Montironi R et al. 2004 WHO classification of the renal tumours of the adults. Eur Urol 2006; 49: 798.

9. Fuhrman SA, Lasky LC and Limas C. Prognostic significance of morphologic parameters in renal cell carcinoma. Am J Surg Pathol 1982; 6: 655.

10. Amin M, Greene F, Edge S et al. The Eighth Edition AJCC Cancer Staging Manual: Continuing to build a bridge from a population-based to a more "personalized" approach to cancer staging. CA Cancer J Clin 2017; 67: 93.

11. Strupler M, Hernest M, Fligny $C$ et al. Second harmonic microscopy to quantify renal interstitial fibrosis and arterial remodeling. J Biomed Opt 2008; 13(5): 054041.

12. Tai DC, Tan N, Xu S et al. Fibro-C-Index: comprehensive, morphology-based quantification of liver fibrosis using second harmonic generation and two-photon microscopy. J Biomed Opt 2009; 14 : 044013.

13. Sun W, Chang S, Tai DC, et al. Nonlinear optical microscopy: use of second harmonic generation and two-photon microscopy for automated quantitative liver fibrosis studies. J Biomed Opt 2008; 13 : 064010.

14. Wu X, Chen G, Qiu J, et al. Visualization of basement membranes in normal breast and breast cancer tissues using multiphoton microscopy. Oncol Lett 2016; 11(6): 3785.

15. Tilbury K and Campagnola PJ: Applications of second-harmonic generation imaging microscopy in ovarian and breast cancer. Perspect Medicin Chem 2015; 7: 21.

16. He K, Zhao L, Huang $X$, et al. Label-free imaging for T staging of gastric carcinoma by multiphoton microscopy. Lasers Med Sci 2018; 33(4): 871.

17. Ling Y, Li C, Feng K, et al. Second harmonic generation (SHG) imaging of cancer heterogeneity in ultrasound guided biopsies of prostate in men suspected with prostate cancer. J Biophotonics 2017; 10(6-7): 911.

18. Brooks $M, M o Q$, Krasnow $R$, et al. Positive association of collagen type I with non-muscle invasive bladder cancer progression. Oncotarget 2016; 7(50): 82609. 
19. Best S, Liu Y, Adib K, et al. Collagen organization of renal cell carcinoma differs between low and high grade tumours. BMC Cancer 2019; 19: 490.

20. Jain $M$, Robinson B, Aggarwal A, et al. Multiphoton microscopy for rapid histopathological evaluation of kidney tumours. BJU Int 2016; 118: 118.

21. Cho S, Lee J, Jeon S, et al. A prospective, multicenter analysis of pseudocapsule characteristics: Do all stages of renal cell carcinoma have complete pseudocapsules? Urol Oncol 2017; 35: 370.

22. Kim M, Choi W, Jeong C, et al. Differences in peritumoral pseudocapsule characteristics according to clinicopathological factors in clinical T1a renal tumours. Int J Clin Exp Pathol 2015; 1: 11323.

23. Provenzano P, Eliceiri K, Campbell J, et al. Collagen reorganization at the tumour-stromal interface facilitates local invasion. BMC Med 2006; 26: 38.

24. Wang L, Feng J, Alvarez $\mathrm{H}$, et al. Critical histologic appraisal of the pseudocapsule of small renal tumours. Virchows Arch 2015; 467(3): 311.

\section{Figures}

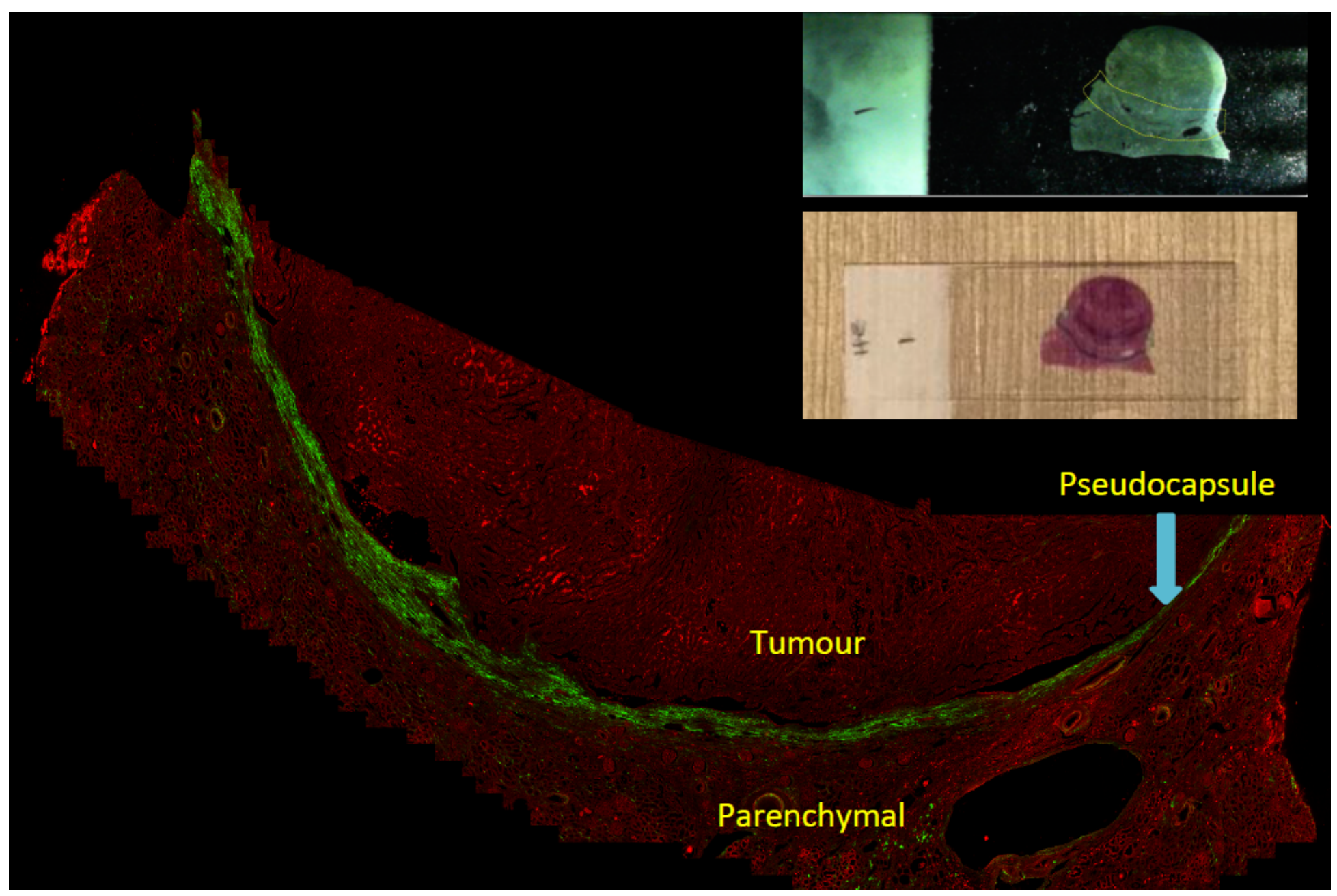

\section{Figure 1}

MPM imaging of RCC Pseudocapsule at 2X Objective 


\section{TPE only}

\section{Tumour}

\section{Parenchymal}

\section{SHG only}

\section{Collagen} profile $=$ densely packed and "aligned"

Normal region $=$ collagen fibers appears short loosely packed
7 Tumour region = little/ void of collagen
Pseudocapsule

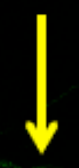

Figure 2

Comparison of TPE and SHG Imaging Signals at 2X Objective 


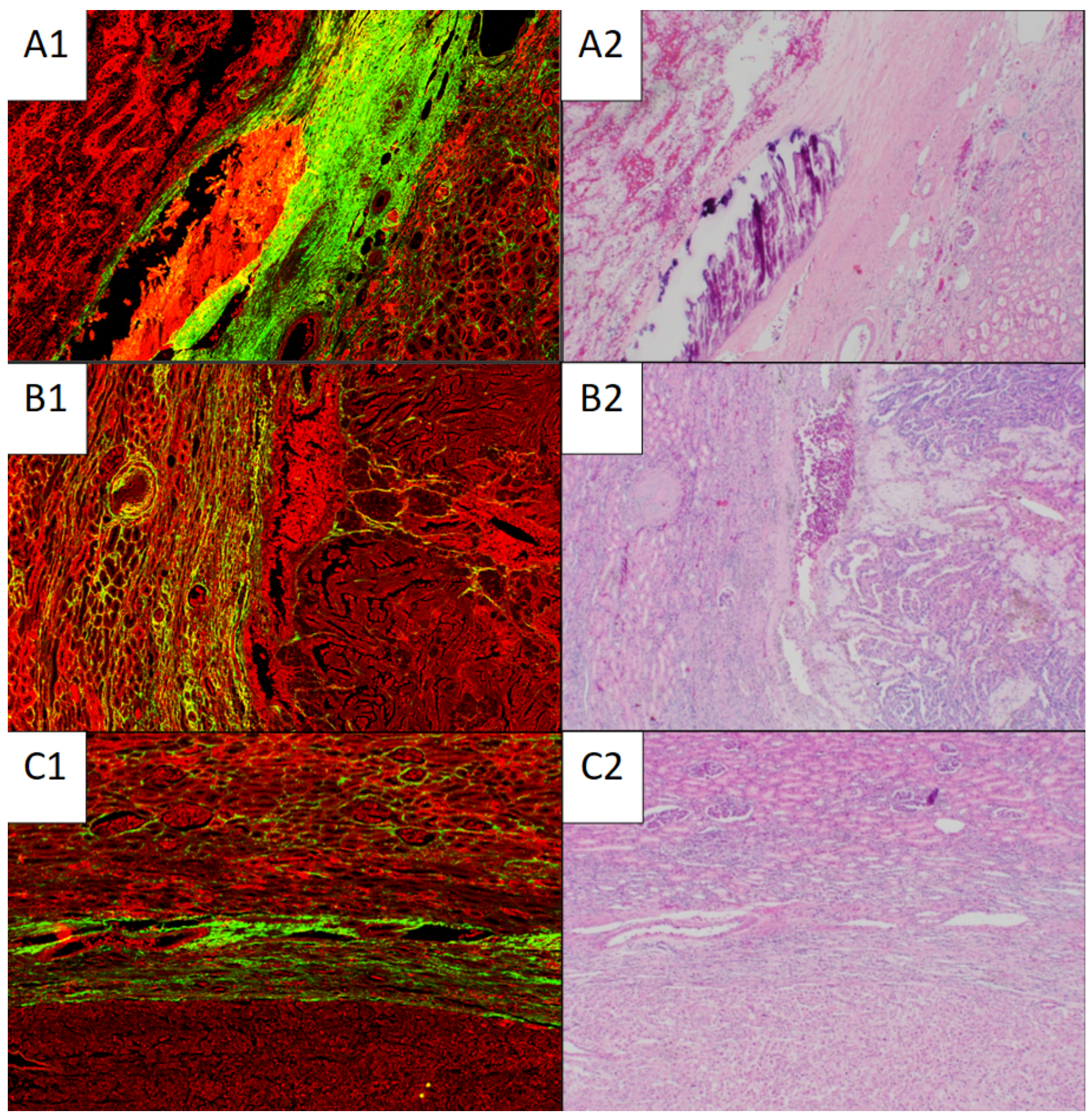

\section{Figure 3}

MPM Images of RCC Pseudocapsules and their corresponding Hematoxylin and Eosin (H\&E) image at 20X magnification. (A1) and (A2) show a Clear Cell RCC Pseudocapsule, (B1) and (B2) show a Papillary RCC Pseudocapsule, and (C1) and (C2) show a Chromophobe RCC Pseudocapsule. Collagen at the pseudocapsule is stained fluorescent green on MPM. 


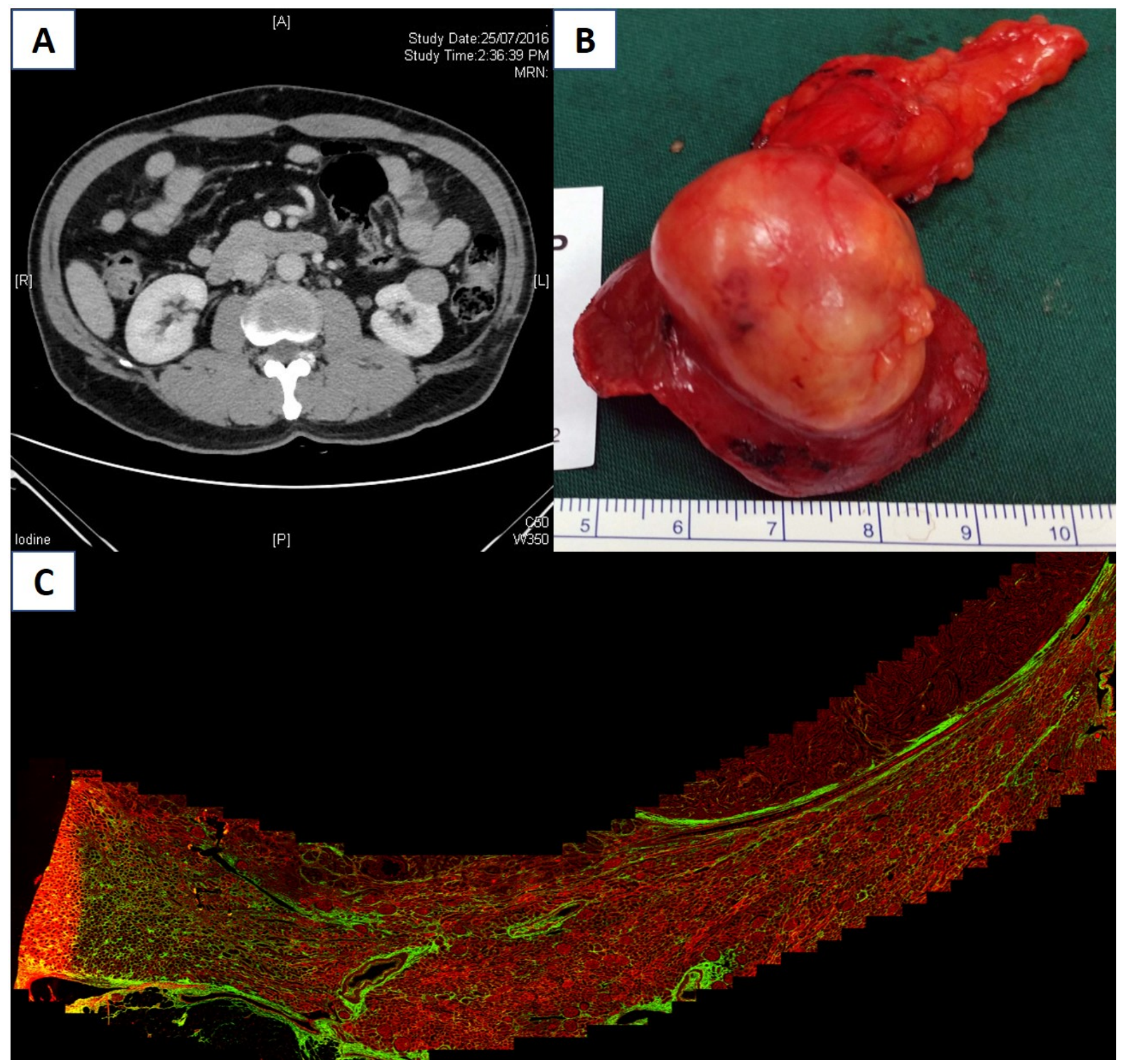

Figure 4

CT Scan (A) Image, Tumor Specimen (B) and MPM Imaging at 2X objective (C) of a pT1a Papillary RCC. Collagen at the pseudocapsule is stained fluorescent green on MPM. 


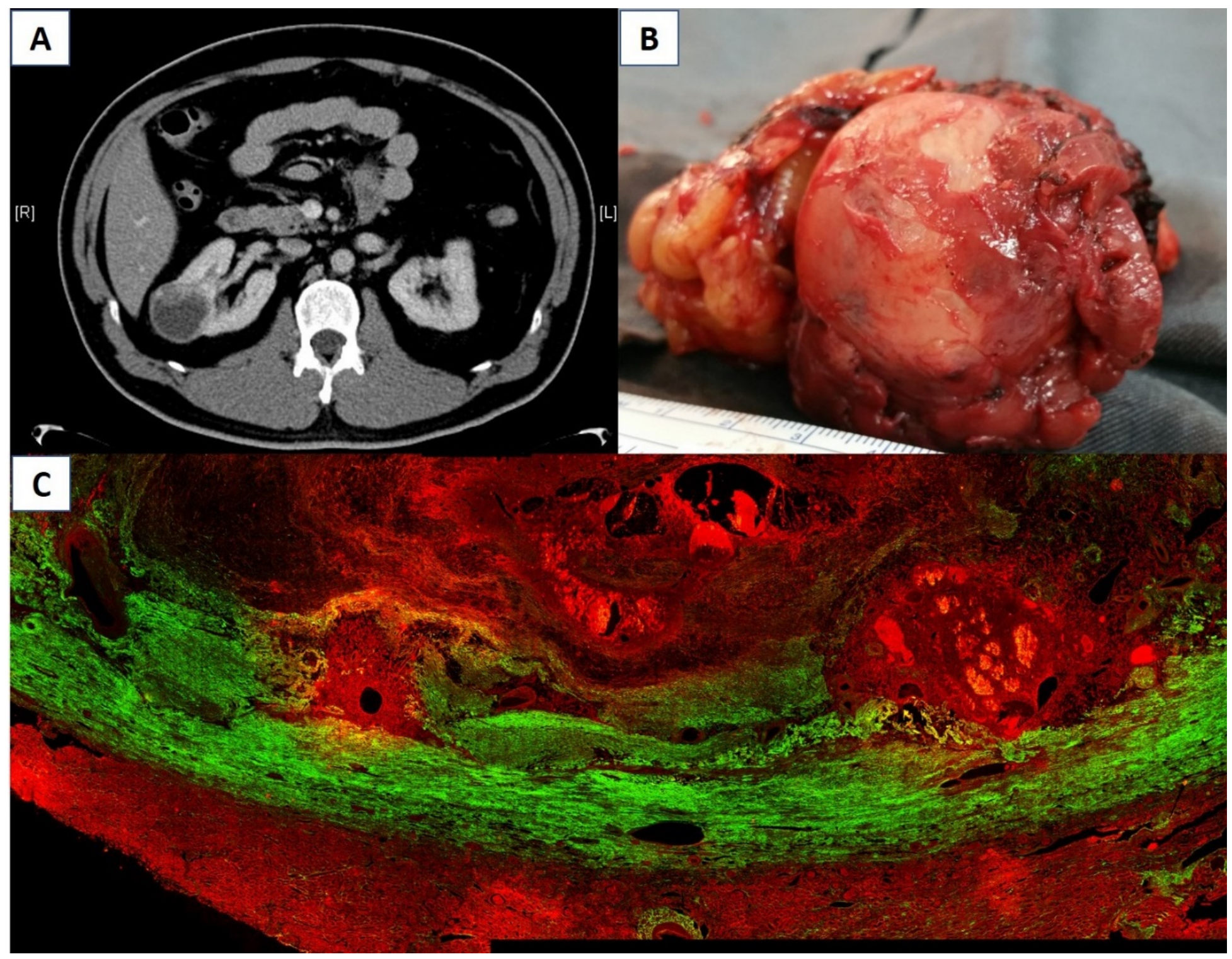

Figure 5

CT Scan (A) Image, Tumor Specimen (B) and MPM Imaging at 2X objective (C) of a pT1b Clear Cell RCC. Collagen at the pseudocapsule is stained fluorescent green on MPM. 